\title{
Research Article \\ On the Stability of a General Mixed Additive-Cubic Functional Equation in Random Normed Spaces
}

\author{
Tian Zhou Xu, ${ }^{1}$ John Michael Rassias, ${ }^{2}$ and Wan $\mathbf{X i n} \mathbf{X u}^{3}$ \\ ${ }^{1}$ Department of Mathematics, School of Science, Beijing Institute of Technology, Beijing 100081, China \\ ${ }^{2}$ Pedagogical Department E.E., Section of Mathematics and Informatics, National and \\ Kapodistrian University of Athens, 4, Agamemnonos Str., Aghia Paraskevi, 15342 Athens, Greece \\ ${ }^{3}$ School of Communication and Information Engineering, University of Electronic Science and \\ Technology of China, Chengdu 611731, China
}

Correspondence should be addressed to Tian Zhou Xu, xutianzhou@bit.edu.cn

Received 6 June 2010; Revised 1 August 2010; Accepted 23 August 2010

Academic Editor: Radu Precup

Copyright (c) 2010 Tian Zhou Xu et al. This is an open access article distributed under the Creative Commons Attribution License, which permits unrestricted use, distribution, and reproduction in any medium, provided the original work is properly cited.

We prove the generalized Hyers-Ulam stability of the following additive-cubic equation $f(k x+$ $y)+f(k x-y)=k f(x+y)+k f(x-y)+2 f(k x)-2 k f(x)$ in the setting of random normed spaces.

\section{Introduction}

A basic question in the theory of functional equations is as follows: when is it true that a function, which approximately satisfies a functional equation, must be close to an exact solution of the equation?

If the problem accepts a unique solution, we say the equation is stable (see [1]). The first stability problem concerning group homomorphisms was raised by Ulam [2] in 1940 and affirmatively solved by Hyers [3]. The result of Hyers was generalized by Rassias [4] for approximate linear mappings by allowing the Cauchy difference operator $C D f(x, y)=$ $f(x+y)-[f(x)+f(y)]$ to be controlled by $\epsilon\left(\|x\|^{p}+\|y\|^{p}\right)$. In 1994, a generalization of Rassias' theorem was obtained by Găvruţa [5], who replaced $\epsilon\left(\|x\|^{p}+\|y\|^{p}\right)$ by a general control function $\varphi(x, y)$ in the spirit of Th. M. Rassias' approach. The stability problems of several functional equations have been extensively investigated by a number of authors, and there are many interesting results concerning this problem (see, e.g., [6-12] and references therein). In addition, J. M. Rassias et al. [13-16] generalized the Hyers stability result by introducing two weaker conditions controlled by the Ulam-Gavruta-Rassias (or UGR) product of different powers of norms and the JM Rassias (or JMR) mixed product-sum of powers of norms, respectively. 
The theory of random normed spaces (RN-spaces) is important as a generalization of deterministic result of linear normed spaces and also in the study of random operator equations. The RN-spaces may also provide us the appropriate tools to study the geometry of nuclear physics and have important application in quantum particle physics (see [17] and the references therein). The generalized Hyers-Ulam stability of different functional equations in random normed spaces, fuzzy normed spaces, and non-Archimedean fuzzy normed spaces has been recently studied in [14-28].

Najati and Eskandani [29] established the general solution and investigated the UlamHyers stability of the following functional equation.

$$
f(2 x+y)+f(2 x-y)=2 f(x+y)+2 f(x-y)+2 f(2 x)-4 f(x)
$$

with $f(0)=0$ in the quasi-Banach spaces. It is easy to see that the mapping $f(x)=a x^{3}+b x$ is a solution of the functional equation (1.1), which is called a mixed additive-cubic functional equation, and every solution of the mixed additive-cubic functional equation is said to be a mixed additive-cubic mapping.

In [14-16], we considered the following general mixed additive-cubic functional equation:

$$
f(k x+y)+f(k x-y)=k f(x+y)+k f(x-y)+2 f(k x)-2 k f(x) .
$$

It is easy to show that the function $f(x)=a x^{3}+b x$ satisfies the functional equation (1.2). We observe that in case $k=2$ (1.2) yields mixed additive-cubic equation (1.1). Therefore, (1.2) is a generalized form of the mixed additive-cubic equation.

In the present paper, we first prove a theorem on stability of equation $g(a x)=$ $a^{s} g(x)(a, s \in \mathbb{N}, a \geq 2)$ in random normed spaces and derive from it results on stability of equation $f(4 x)=10 f(2 x)-16 f(x)$. Next, use those results to establish Ulam-Hyers stability for the general mixed additive-cubic functional equation (1.2) in the setting of random normed spaces. In this way some results will be obtained on stability of the linear functional equations also for the random normed spaces, which correspond, for example, to the papers [30-33].

\section{Preliminaries}

In the sequel we adopt the usual terminology, notations and conventions of the theory of random normed spaces, as in $[17,28]$. Throughout this paper, the space of all probability distribution functions is denoted by

$$
\begin{aligned}
& \Delta^{+}=\{F: \mathbb{R} \cup\{-\infty,+\infty\} \rightarrow[0,1]: F \text { is left-continuous and nondecreasing on } \mathbb{R} \\
& \text { and } F(0)=0, F(+\infty)=1\},
\end{aligned}
$$

and the subset $D^{+} \subseteq \Delta^{+}$is the set $D^{+}=\left\{F \in \Delta^{+}: l^{-} F(+\infty)=1\right\}$, where $l^{-} f(x)$ denotes the left limit of the function $f$ at the point $x$. The space $\Delta^{+}$is partially ordered by the usual pointwise 
ordering of functions, that is, $F \leq G$ if and only if $F(t) \leq G(t)$ for all $t \in \mathbb{R}$. The maximal element for $\Delta^{+}$in this order is the distribution function given by

$$
\varepsilon_{0}(t)= \begin{cases}0, & \text { if } t \leq 0 \\ 1, & \text { if } t>0\end{cases}
$$

Definition 2.1 (see $[17,28]$ ). A function $\tau:[0,1] \times[0,1] \rightarrow[0,1]$ is a continuous triangular norm (briefly, a continuous $t$-norm) if $\tau$ satisfies the following conditions:

(a) $\tau$ is commutative and associative;

(b) $\tau$ is continuous;

(c) $\tau(a, 1)=a$ for all $a \in[0,1]$;

(d) $\tau(a, b) \leq \tau(c, d)$ whenever $a \leq c$ and $b \leq d$ for all $a, b, c, d \in[0,1]$.

Typical examples of continuous $t$-norms are $\tau_{P}(a, b)=a b, \tau_{M}(a, b)=\min (a, b)$ and $\tau_{L}(a, b)=\max (a+b-1,0)$ (the Lukasiewicz $t$-norm).

Now, if $\tau$ is a $t$-norm and $\left\{x_{i}\right\}$ is a given sequence of numbers in $[0,1]$, we define a sequence $\tau^{n}$ recursively by $\tau_{i=1}^{1} x_{1}=x_{1}$ and $\tau_{i=1}^{n} x_{i}=\tau\left(\tau_{i=1}^{n-1} x_{i}, x_{n}\right)$ for all $n \geq 2$. $\tau_{i=n}^{\infty} x_{i}$ is defined as $\tau_{i=1}^{\infty} x_{n+i}$.

Definition 2.2 (see $[17,28]$ ). A random normed space (briefly, RN-space) is a triple $(X, \mu, \tau)$, where $X$ is a vector space, $\tau$ is a continuous $t$-norm, and $\mu$ is a mapping from $X$ into $D^{+}$such that the following conditions hold:

$$
\begin{aligned}
& \text { (RN1) } \mu_{x}(t)=\varepsilon_{0}(t) \text { for all } t>0 \text { if and only if } x=0 \text {; } \\
& \text { (RN2) } \mu_{\alpha x}(t)=\mu_{x}(t /|\alpha|) \text { for all } x \text { in } X, \alpha \neq 0 \text { and all } t \geq 0 \text {; } \\
& \text { (RN3) } \mu_{x+y}(t+s) \geq \tau\left(\mu_{x}(t), \mu_{y}(s)\right) \text { for all } x, y \in X \text { and all } t, s \geq 0 .
\end{aligned}
$$

Example 2.3. Let $(X,\|\cdot\|)$ be a normed space. For all $x \in X$ and $t>0$, consider $\mu_{x}(t)=$ $t /(t+\|x\|)$. Then $\left(X, \mu, \tau_{M}\right)$ is a random normed space, where $\tau_{M}$ is the minimum $t$-norm. This space is called the induced random normed space.

Definition 2.4. Let $(X, \mu, \tau)$ be an $\mathrm{RN}$-space.

(1) A sequence $\left\{x_{n}\right\}$ in $X$ is said to be convergent to a point $x \in X$ if, for every $t>0$ and $\varepsilon>0$, there exists a positive integer $N$ such that $\mu_{x_{n}-x}(t)>1-\varepsilon$ whenever $n \geq N$.

(2) A sequence $\left\{x_{n}\right\}$ in $X$ is called a Cauchy sequence if, for every $t>0$ and $\varepsilon>0$, there exists a positive integer $N$ such that $\mu_{x_{n}-x_{m}}(t)>1-\varepsilon$ whenever $n \geq m \geq N$.

(3) An RN-space $(X, \mu, \tau)$ is said to be complete if and only if every Cauchy sequence in $X$ is convergent to a point in $X$. 


\section{On the Stability of a General Mixed Additive-Cubic Equation in RN-Spaces}

Theorem 3.1. Let $s, a \in \mathbb{N}$ with $a \geq 2, X$ be a linear space, $\left(Y, \mu, \tau_{M}\right)$ be a complete $R N$-space, and $g: X \rightarrow Y$ be a mapping for which there is $\psi: X \rightarrow D^{+}$such that

$$
\mu_{g(a x)-a^{s} g(x)}(t) \geq \psi_{x}(t)
$$

for all $x \in X$ and $t>0$. If for some $0<\alpha<a^{s}$,

$$
\psi_{a x}(t) \geq \psi_{x}\left(\frac{t}{\alpha}\right)
$$

for all $x \in X$ and $t>0$, then there exists a uniquely determined mapping $G: X \rightarrow Y$ such that $G(a x)=a^{s} G(x)$ and

$$
\mu_{g(x)-G(x)}(t) \geq \psi_{x}\left(\frac{\left(a^{s}-\alpha\right) t}{2}\right)
$$

for all $x \in X$ and $t>0$.

Proof. Replacing $x$ by $a^{i} x$ in (3.1) and using (3.2), we get

$$
\mu_{\left(g\left(a^{i+1} x\right) / a^{s(i+1)}\right)-\left(g\left(a^{i} x\right) / a^{s i}\right)}\left(\frac{\alpha^{i} t}{a^{s(i+1)}}\right) \geq \psi_{x}(t)
$$

for all $x \in X, i \in \mathbb{N}$, and $t>0$. It follows that

$$
\begin{aligned}
& \mu_{\left(g\left(a^{n} x\right) / a^{s n}\right)-\left(g\left(a^{m} x\right) / a^{s m}\right)}\left(\sum_{i=m}^{n-1} \frac{\alpha^{i} t}{a^{s(i+1)}}\right) \\
& =\mu_{\sum_{i=m}^{n-1}\left(\left(g\left(a^{i+1} x\right) / a^{s(i+1)}\right)-\left(g\left(a^{i} x\right) / a^{s i}\right)\right)}\left(\sum_{i=m}^{n-1} \frac{\alpha^{i} t}{a^{s(i+1)}}\right) \geq \psi_{x}(t)
\end{aligned}
$$

for all $x \in X, t>0$ and all nonnegative integers $n$ and $m$ with $n>m$. Hence

$$
\mu_{\left(g\left(a^{n} x\right) / a^{s n}\right)-\left(g\left(a^{m} x\right) / a^{s m}\right)}(t) \geq \psi_{x}\left(t / \sum_{i=m}^{n-1} \frac{\alpha^{i}}{a^{s(i+1)}}\right)
$$

for all $x \in X, t>0$, and $m, n \in \mathbb{N}$ with $n>m$. As $0<\alpha<a^{s}$ and $\sum_{i=0}^{\infty}\left(\alpha^{i} / a^{s(i+1)}\right)<\infty$, the right hand side of the inequality tends to 1 as $m$ tend to infinity. Then the sequence $\left\{g\left(a^{n} x\right) / a^{s n}\right\}$ is a Cauchy sequence in $\left(Y, \mu, \tau_{M}\right)$. Since $\left(Y, \mu, \tau_{M}\right)$ is a complete $\mathrm{RN}$-space, this sequence converges to some point $G(x) \in Y$. Therefore, we may define $G(x):=\lim _{n \rightarrow \infty} g\left(a^{n} x\right) / a^{s n}$ for all $x \in X$. Fix $x \in X$, and put $m=0$ in (3.6). Then we obtain

$$
\mu_{\left(g\left(a^{n} x\right) / a^{s n}\right)-g(x)}(t) \geq \psi_{x}\left(t / \sum_{i=0}^{n-1} \frac{\alpha^{i}}{a^{s(i+1)}}\right),
$$


and so, by (RN3), we have

$$
\begin{aligned}
\mu_{G(x)-g(x)}(t) & \geq \tau_{M}\left(\mu_{G(x)-\left(g\left(a^{n} x\right) / a^{s n}\right)}\left(\frac{t}{2}\right), \mu_{\left(g\left(a^{n} x\right) / a^{s n}\right)-g(x)}\left(\frac{t}{2}\right)\right) \\
& \geq \tau_{M}\left(\mu_{G(x)-\left(g\left(a^{n} x\right) / a^{s n}\right)}\left(\frac{t}{2}\right), \psi_{x}\left(t / \sum_{i=0}^{n-1} \frac{2 \alpha^{i}}{a^{s(i+1)}}\right)\right)
\end{aligned}
$$

for every $t>0$. Taking the limit as $n \rightarrow \infty$ in (3.8), by $G(x)=\lim _{n \rightarrow \infty} g\left(a^{n} x\right) / a^{s n}$, we get (3.3).

To prove the uniqueness of the mapping $G$, assume that there exists another mapping $H: X \rightarrow Y$ which satisfies (3.3) and $H(a x)=a^{s} H(x)$ for all $x \in X$. Fix $x \in X$. Clearly, $G\left(a^{n} x\right)=a^{s n} G(x)$, and $H\left(a^{n} x\right)=a^{s n} H(x)$ for all $n \in \mathbb{N}$. It follows from (3.2) and (3.3) that

$$
\begin{aligned}
\mu_{G(x)-H(x)}(t) & \geq \tau_{M}\left(\mu_{\left(G\left(a^{n} x\right) / a^{s n}\right)-\left(g\left(a^{n} x\right) / a^{s n}\right)}\left(\frac{t}{2}\right), \mu_{\left(g\left(a^{n} x\right) / a^{s n}\right)-\left(H\left(a^{n} x\right) / a^{s n}\right)}\left(\frac{t}{2}\right)\right) \\
& \geq \psi_{x}\left(\frac{\left(a^{s}-\alpha\right) a^{s n} t}{4 \alpha^{n}}\right) .
\end{aligned}
$$

Since $\lim _{n \rightarrow \infty}\left(a^{s}-\alpha\right) a^{s n} t /\left(4 \alpha^{n}\right)=\infty$, we get $\lim _{n \rightarrow \infty} \psi_{x}\left(\left(a^{s}-\alpha\right) a^{s n} t /\left(4 \alpha^{n}\right)\right)=1$. Therefore, it follows from (3.9) that $\mu_{G(x)-H(x)}(t)=1$ for all $t>0$, and so $G=H$. This completes the proof.

Corollary 3.2. Let $s \in\{1,3\}$ be fixed, $X$ be a linear space, $\left(Y, \mu, \tau_{M}\right)$ be a complete $R N$-space, and $f: X \rightarrow Y$ be a mapping for which there is $\psi: X \rightarrow D^{+}$such that

$$
\mu_{f(4 x)-10 f(2 x)+16 f(x)}(t) \geq \psi_{x}(t)
$$

for all $x \in X$ and $t>0$. If for some $0<\alpha<2^{s}, \psi_{2 x}(t) \geq \psi_{x}(t / \alpha)$ for all $x \in X$ and $t>0$, then there exists a uniquely determined mapping $F_{S}: X \rightarrow Y$ such that $F_{S}(2 x)=2^{s} F_{S}(x)$ and

$$
\mu_{f(2 x)-2^{3 / s} f(x)-F_{s}(x)}(t) \geq \psi_{x}\left(\frac{\left(2^{s}-\alpha\right) t}{2}\right)
$$

for all $x \in X$ and $t>0$.

Theorem 3.3. Let $X$ be a linear space, $\left(Z, \mu^{\prime}, \tau_{M}\right)$ be an $R N$-space, $\left(Y, \mu, \tau_{M}\right)$ be a complete $R N$ space, and $f: X \rightarrow Y$ be a mapping with $f(0)=0$ for which there is $\varphi: X \times X \rightarrow Z$ such that

$$
\mu_{f(k x+y)+f(k x-y)-k f(x+y)-k f(x-y)-2 f(k x)+2 k f(x)}(t) \geq \mu_{\varphi(x, y)}^{\prime}(t)
$$

for all $x, y \in X$ and $t>0$. If for some $0<\alpha<2$,

$$
\mu_{\varphi(2 x, 2 y)}^{\prime}(t) \geq \mu_{\alpha \varphi(x, y)}^{\prime}(t)
$$


for all $x, y \in X$ and $t>0$, then there exists a unique additive mapping $A: X \rightarrow Y$ such that

$$
\mu_{f(2 x)-8 f(x)-A(x)}(t) \geq \psi_{x}\left(\frac{(2-\alpha)\left(k^{3}-k\right) t}{2}\right)
$$

for all $x \in X$ and $t>0$, where

$$
\begin{aligned}
\psi_{x}(t):=\left(\tau_{M}\right)_{i=1}^{32}( & \mu_{\varphi(x / 2,(2 k+1) x / 2)}^{\prime}\left(\frac{t}{384 k}\right), \mu_{\varphi(x / 2,(2 k-1) x / 2)}^{\prime}\left(\frac{t}{384 k}\right), \\
& \mu_{\varphi(x / 2,3 k x / 2)}^{\prime}\left(\frac{t}{384}\right), \mu_{\varphi(0,(3 k-1) x / 2)}^{\prime}\left(\frac{(k-1) t}{384 k}\right), \mu_{\varphi(x, x)}^{\prime}\left(\frac{t}{96 k^{2}}\right), \\
& \mu_{\varphi(x / 2, k x / 2)}^{\prime}\left(\frac{t}{96}\right), \mu_{\varphi(0,(k+1) x / 2)}^{\prime}\left(\frac{(k-1) t}{96 k}\right), \mu_{\varphi(0,(k-1) x)}^{\prime}\left(\frac{(k-1) t}{96 k^{2}}\right), \\
& \mu_{\varphi(0, k x)}^{\prime}\left(\frac{t}{96(k+1)}\right), \mu_{\varphi(x,(k+1) x)}^{\prime}\left(\frac{t}{128}\right), \mu_{\varphi(x,(k-1) x)}^{\prime}\left(\frac{t}{128}\right), \\
& \mu_{\varphi(0, x)}^{\prime}\left(\frac{(k-1) t}{128}\right), \mu_{\varphi(0, k x)}^{\prime}\left(\frac{(k-1) t}{128 k}\right), \mu_{\varphi(2 x, x)}^{\prime}\left(\frac{t}{32}\right), \mu_{\varphi(2 x, k x)}^{\prime}\left(\frac{t}{16}\right), \\
& \mu_{\varphi(x,(2 k-1) x)}^{\prime}\left(\frac{t}{56}\right), \mu_{\varphi(x,(2 k+1) x)}^{\prime}\left(\frac{t}{56}\right), \mu_{3 x, x}^{\prime}\left(\frac{t}{56}\right), \mu_{\varphi(x, x)}^{\prime}\left(\frac{t}{56}\right), \\
& \mu_{\varphi(0,(k+1) x)}^{\prime}\left(\frac{(k-1) t}{56}\right), \mu_{\varphi(0,(k-1) x)}^{\prime}\left(\frac{(k-1) t}{56}\right), \mu_{\varphi(0,2 k x)}^{\prime}\left(\frac{(k-1) t}{56 k}\right), \\
& \mu_{\varphi(x,(2 k+1) x)}^{\prime}\left(\frac{t}{384 k}\right), \mu_{\varphi(x,(2 k-1) x)}^{\prime}\left(\frac{t}{384 k}\right), \mu_{\varphi(x, 3 k x)}^{\prime}\left(\frac{t}{384}\right), \\
& \mu_{\varphi(0,(3 k-1) x)}^{\prime}\left(\frac{(k-1) t}{384 k}\right), \mu_{\varphi(2 x, 2 x)}^{\prime}\left(\frac{t}{96 k^{2}}\right), \mu_{\varphi(x, k x)}^{\prime}\left(\frac{t}{96}\right), \\
& \mu_{\varphi(0,(k+1) x)}^{\prime}\left(\frac{(k-1) t}{96 k}\right), \mu_{\varphi(0,2(k-1) x)}^{\prime}\left(\frac{(k-1) t}{96 k^{2}}\right), \\
& \left.\mu_{\varphi(0,2 k x)}^{\prime}\left(\frac{t}{96(k+1)}\right), \mu_{\varphi(2 x, 2 k x)}^{\prime}\left(\frac{t}{16}\right)\right) . \\
&
\end{aligned}
$$

Proof. Letting $x=0$ in (3.12), we get

$$
\mu_{f(y)+f(-y)}(t) \geq \mu_{\varphi(0, y)}^{\prime}((k-1) t)
$$

for all $y \in X$ and $t>0$. Putting $y=x$ in (3.12), we have

$$
\mu_{f((k+1) x)+f((k-1) x)-k f(2 x)-2 f(k x)+2 k f(x)}(t) \geq \mu_{\varphi(x, x)}^{\prime}(t)
$$


Journal of Inequalities and Applications

for all $x \in X$ and $t>0$. Replacing $x$ by $2 x$ in (3.17), we obtain

$$
\mu_{f(2(k+1) x)+f(2(k-1) x)-k f(4 x)-2 f(2 k x)+2 k f(2 x)}(t) \geq \mu_{\varphi(2 x, 2 x)}^{\prime}(t)
$$

for all $x \in X$ and $t>0$. Letting $y=k x$ in (3.12), we get

$$
\mu_{f(2 k x)-k f((k+1) x)-k f(-(k-1) x)-2 f(k x)+2 k f(x)}(t) \geq \mu_{\varphi(x, k x)}^{\prime}(t)
$$

for all $x \in X$ and $t>0$. Letting $y=(k+1) x$ in (3.12), we have

$$
\mu_{f((2 k+1) x)+f(-x)-k f((k+2) x)-k f(-k x)-2 f(k x)+2 k f(x)}(t) \geq \mu_{\varphi(x,(k+1) x)}^{\prime}(t)
$$

for all $x \in X$ and $t>0$. Letting $y=(k-1) x$ in (3.12), we have

$$
\mu_{f((2 k-1) x)-(k+2) f(k x)-k f(-(k-2) x)+(2 k+1) f(x)}(t) \geq \mu_{\varphi(x,(k-1) x)}^{\prime}(t)
$$

for all $x \in X$ and $t>0$. Replacing $x$ and $y$ by $2 x$ and $x$ in (3.12), respectively, we get

$$
\mu_{f((2 k+1) x)+f((2 k-1) x)-2 f(2 k x)-k f(3 x)+2 k f(2 x)-k f(x)}(t) \geq \mu_{\varphi(2 x, x)}^{\prime}(t)
$$

for all $x \in X$ and $t>0$. Replacing $x$ and $y$ by $3 x$ and $x$ in (3.12), respectively, we get

$$
\mu_{f((3 k+1) x)+f((3 k-1) x)-2 f(3 k x)-k f(4 x)-k f(2 x)+2 k f(3 x)}(t) \geq \mu_{\varphi(3 x, x)}^{\prime}(t)
$$

for all $x \in X$ and $t>0$. Replacing $x$ and $y$ by $2 x$ and $k x$ in (3.12), respectively, we have

$$
\mu_{f(3 k x)+f(k x)-k f((k+2) x)-k f(-(k-2) x)-2 f(2 k x)+2 k f(2 x)}(t) \geq \mu_{\varphi(2 x, k x)}^{\prime}(t)
$$

for all $x \in X$ and $t>0$. Setting $y=(2 k+1) x$ in (3.12), we have

$$
\mu_{f((3 k+1) x)+f(-(k+1) x)-k f(2(k+1) x)-k f(-2 k x)-2 f(k x)+2 k f(x)}(t) \geq \mu_{\varphi(x,(2 k+1) x)}^{\prime}(t)
$$

for all $x \in X$ and $t>0$. Letting $y=(2 k-1) x$ in (3.12), we have

$$
\mu_{f((3 k-1) x)+f(-(k-1) x)-k f(-2(k-1) x)-k f(2 k x)-2 f(k x)+2 k f(x)}(t) \geq \mu_{\varphi(x,(2 k-1) x)}^{\prime}(t)
$$

for all $x \in X$ and $t>0$. Letting $y=3 k x$ in (3.12), we have

$$
\mu_{f(4 k x)+f(-2 k x)-k f((3 k+1) x)-k f(-(3 k-1) x)-2 f(k x)+2 k f(x)}(t) \geq \mu_{\varphi(x, 3 k x)}^{\prime}(t)
$$


for all $x \in X$ and $t>0$. By (3.16), (3.17), (3.23), (3.25), and (3.26), we get

$$
\begin{aligned}
& \mu_{k f(2(k+1) x)+k f(-2(k-1) x)+6 f(k x)-2 f(3 k x)-k f(4 x)+2 k f(3 x)-6 k f(x)}(t) \\
& \geq\left(\tau_{M}\right)_{i=1}^{7}\left(\mu_{\varphi(x,(2 k-1) x)}^{\prime}\left(\frac{t}{7}\right), \mu_{\varphi(\mathrm{x},(2 k+1) x)}^{\prime}\left(\frac{t}{7}\right), \mu_{\varphi(3 x, x)}^{\prime}\left(\frac{t}{7}\right), \mu_{\varphi(x, x)}^{\prime}\left(\frac{t}{7}\right),\right. \\
& \left.\mu_{\varphi(0,(k+1) x)}^{\prime}\left(\frac{(k-1) t}{7}\right), \mu_{\varphi(0,(k-1) x)}^{\prime}\left(\frac{(k-1) t}{7}\right), \mu_{\varphi(0,2 k x)}^{\prime}\left(\frac{(k-1) t}{7 k}\right)\right)
\end{aligned}
$$

for all $x \in X$ and $t>0$. By (3.16), (3.20), and (3.21), we have

$$
\begin{aligned}
& \mu_{f((2 k+1) x)+f((2 \mathrm{k}-1) x)-k f((k+2) x)-k f(-(k-2) x)-4 f(k x)+4 k f(x)}(t) \\
& \quad \geq\left(\tau_{M}\right)_{i=1}^{4}\left(\mu_{\varphi(x,(k+1) x)}^{\prime}\left(\frac{t}{4}\right), \mu_{\varphi(x,(k-1) x)}^{\prime}\left(\frac{t}{4}\right), \mu_{\varphi(0, x)}^{\prime}\left(\frac{(k-1) t}{4}\right), \mu_{\varphi(0, k x)}^{\prime}\left(\frac{(k-1) t}{4 k}\right)\right)
\end{aligned}
$$

for all $x \in X$ and $t>0$. It follows from (3.22) and (3.29) that

$$
\begin{gathered}
\mu_{k f((k+2) x)+k f(-(k-2) x)-2 f(2 k x)+4 f(k x)-k f(3 x)+2 k f(2 x)-5 k f(x)}(t) \\
\geq\left(\mathcal{C}_{M}\right)_{i=1}^{5}\left(\mu_{\varphi(x,(k+1) x)}^{\prime}\left(\frac{t}{8}\right), \mu_{\varphi(x,(k-1) x)}^{\prime}\left(\frac{t}{8}\right),\right. \\
\left.\mu_{\varphi(0, x)}^{\prime}\left(\frac{(k-1) t}{8}\right), \mu_{\varphi(0, k x)}^{\prime}\left(\frac{(k-1) t}{8 k}\right), \mu_{\varphi(2 x, x)}^{\prime}\left(\frac{t}{2}\right)\right)
\end{gathered}
$$

for all $x \in X$ and $\mathrm{t}>0$. By (3.24) and (3.30), we have

$$
\begin{gathered}
\mu_{f(3 k x)-4 f(2 k x)+5 f(k x)-k f(3 x)+4 k f(2 x)-5 k f(x)}(t) \\
\geq\left(\mathcal{C}_{M}\right)_{i=1}^{6}\left(\mu_{\varphi(x,(k+1) x)}^{\prime}\left(\frac{t}{16}\right), \mu_{\varphi(x,(k-1) x)}^{\prime}\left(\frac{t}{16}\right), \mu_{\varphi(0, x)}^{\prime}\left(\frac{(k-1) t}{16}\right),\right. \\
\left.\mu_{\varphi(0, k x)}^{\prime}\left(\frac{(k-1) t}{16 k}\right), \mu_{\varphi(2 x, x)}^{\prime}\left(\frac{t}{4}\right), \mu_{\varphi(2 x, k x)}^{\prime}\left(\frac{t}{2}\right)\right)
\end{gathered}
$$

for all $x \in X$ and $t>0$. By (3.16) and (3.25)-(3.27), we have

$$
\begin{aligned}
& \mu_{k f(-(k+1) x)-k f(-(k-1) x)-k^{2} f(2(k+1) x)+k^{2} f(-2(k-1) x)+k^{2} f(2 k x)-\left(k^{2}-1\right) f(-2 k x)+f(4 k x)-2 f(k x)+2 k f(x)}(t) \\
& \quad \geq\left(\tau_{M}\right)_{i=1}^{4}\left(\mu_{\varphi(x,(2 k+1) x)}^{\prime}\left(\frac{t}{4 k}\right), \mu_{\varphi(x,(2 k-1) x)}^{\prime}\left(\frac{t}{4 k}\right), \mu_{\varphi(x, 3 k x)}^{\prime}\left(\frac{t}{4}\right), \mu_{\varphi(0,(3 k-1) x)}^{\prime}\left(\frac{(k-1) t}{4 k}\right)\right)
\end{aligned}
$$


for all $x \in X$ and $t>0$. It follows from (3.16), (3.18), (3.19), and (3.32) that

$$
\begin{gathered}
\mu_{f(4 k x)-2 f(2 k x)-k^{3} f(4 x)+2 k^{3} f(2 x)}(t) \\
\geq\left(\tau_{M}\right)_{i=1}^{9}\left(\mu_{\varphi(x,(2 k+1) x)}^{\prime}\left(\frac{t}{24 k}\right), \mu_{\varphi(x,(2 k-1) x)}^{\prime}\left(\frac{t}{24 k}\right), \mu_{\varphi(x, 3 k x)}^{\prime}\left(\frac{t}{24}\right), \mu_{\varphi(0,(3 k-1) x)}^{\prime}\left(\frac{(k-1) t}{24 k}\right),\right. \\
\mu_{\varphi(2 x, 2 x)}^{\prime}\left(\frac{t}{6 k^{2}}\right), \mu_{\varphi(x, k x)}^{\prime}\left(\frac{t}{6}\right), \mu_{\varphi(0,(k+1) x)}^{\prime}\left(\frac{(k-1) t}{6 k}\right), \\
\left.\mu_{\varphi(0,2(k-1) x)}^{\prime}\left(\frac{(k-1) t}{6 k^{2}}\right), \mu_{\varphi(0,2 k x)}^{\prime}\left(\frac{t}{6(k+1)}\right)\right)
\end{gathered}
$$

for all $x \in X$ and $t>0$. Hence

$$
\begin{aligned}
& \mu_{f(2 k x)-2 f(k x)-k^{3} f(2 x)+2 k^{3} f(x)}(t) \\
& \geq\left(\tau_{M}\right)_{i=1}^{9}\left(\mu_{\varphi(x / 2,(2 k+1) x / 2)}^{\prime}\left(\frac{t}{24 k}\right), \mu_{\varphi(x / 2,(2 k-1) x / 2)}^{\prime}\left(\frac{t}{24 k}\right), \mu_{\varphi(x / 2,3 k x / 2)}^{\prime}\left(\frac{t}{24}\right),\right. \\
& \mu_{\varphi(0,(3 k-1) x / 2)}^{\prime}\left(\frac{(k-1) t}{24 k}\right), \mu_{\varphi(x, x)}^{\prime}\left(\frac{t}{6 k^{2}}\right), \mu_{\varphi(x / 2, k x / 2)}^{\prime}\left(\frac{t}{6}\right), \\
& \left.\mu_{\varphi(0,(k+1) x / 2)}^{\prime}\left(\frac{(k-1) t}{6 k}\right), \mu_{\varphi(0,(k-1) x)}^{\prime}\left(\frac{(k-1) t}{6 k^{2}}\right), \mu_{\varphi(0, k x)}^{\prime}\left(\frac{t}{6(k+1)}\right)\right)
\end{aligned}
$$

for all $x \in X$ and $t>0$. By (3.19), we have

$$
\mu_{f(4 k x)-k f(2(k+1) x)-k f(-2(k-1) x)-2 f(2 k x)+2 k f(2 x)}(t) \geq \mu_{\varphi(2 x, 2 k x)}^{\prime}(t)
$$

for all $x \in X$ and $t>0$. From (3.33) and (3.35), we have

$\mu_{k f(2(k+1) x)+k f(-2(k-1) x)-k^{3} f(4 x)+\left(2 k^{3}-2 k\right) f(2 x)}(t)$

$$
\begin{gathered}
\geq\left(\tau_{M}\right)_{i=1}^{10}\left(\mu_{\varphi(x,(2 k+1) x)}^{\prime}\left(\frac{t}{48 k}\right), \mu_{\varphi(x,(2 k-1) x)}^{\prime}\left(\frac{t}{48 k}\right), \mu_{\varphi(x, 3 k x)}^{\prime}\left(\frac{t}{48}\right), \mu_{\varphi(0,(3 k-1) x)}^{\prime}\left(\frac{(k-1) t}{48 k}\right),\right. \\
\mu_{\varphi(2 x, 2 x)}^{\prime}\left(\frac{t}{12 k^{2}}\right), \mu_{\varphi(x, k x)}^{\prime}\left(\frac{t}{12}\right), \mu_{\varphi(0,(k+1) x)}^{\prime}\left(\frac{(k-1) t}{12 k}\right), \mu_{\varphi(0,2(k-1) x)}^{\prime}\left(\frac{(k-1) t}{12 k^{2}}\right), \\
\left.\mu_{\varphi(0,2 k x)}^{\prime}\left(\frac{t}{12(k+1)}\right), \mu_{\varphi(2 x, 2 k x)}^{\prime}\left(\frac{t}{2}\right)\right)
\end{gathered}
$$


for all $x \in X$ and $t>0$. Also, from (3.28) and (3.36), we get

$$
\begin{aligned}
\mu_{2 f(3 k x)-6 f(k x)+\left(k-k^{3}\right) f(4 x)-2 k f(3 x)+\left(2 k^{3}-2 k\right) f(2 x)+6 k f(x)}(t) \\
\geq\left(\mathcal{乙}_{M}\right)_{i=1}^{17}\left(\mu_{\varphi(x,(2 k-1) x)}^{\prime}\left(\frac{t}{14}\right), \mu_{\varphi(x,(2 k+1) x)}^{\prime}\left(\frac{t}{14}\right),\right. \\
\mu_{\varphi(3 x, x)}^{\prime}\left(\frac{t}{14}\right), \mu_{\varphi(x, x)}^{\prime}\left(\frac{t}{14}\right), \mu_{\varphi(0,(k+1) x)}^{\prime}\left(\frac{(k-1) t}{14}\right), \\
\mu_{\varphi(0,(k-1) x)}^{\prime}\left(\frac{(k-1) t}{14}\right), \mu_{\varphi(0,2 k x)}^{\prime}\left(\frac{(k-1) t}{14 k}\right), \mu_{\varphi(x,(2 k+1) x)}^{\prime}\left(\frac{t}{96 k}\right), \\
\mu_{\varphi(x,(2 k-1) x)}^{\prime}\left(\frac{t}{96 k}\right), \mu_{\varphi(x, 3 k x)}^{\prime}\left(\frac{t}{96}\right), \mu_{\varphi(0,(3 k-1) x)}^{\prime}\left(\frac{(k-1) t}{96 k}\right), \\
\mu_{\varphi(2 x, 2 x)}^{\prime}\left(\frac{t}{24 k^{2}}\right), \mu_{\varphi(x, k x)}^{\prime}\left(\frac{t}{24}\right), \mu_{\varphi(0,(k+1) x)}^{\prime}\left(\frac{(k-1) t}{24 k}\right), \\
\left.\mu_{\varphi(0,2(k-1) x)}^{\prime}\left(\frac{(k-1) t}{24 k^{2}}\right), \mu_{\varphi(0,2 k x)}^{\prime}\left(\frac{t}{24(k+1)}\right), \mu_{\varphi(2 x, 2 k x)}^{\prime}\left(\frac{t}{4}\right)\right)
\end{aligned}
$$

for all $x \in X$ and $t>0$.

On the other hand, it follows from (3.31) and (3.37) that

$$
\begin{gathered}
\mu_{8 f(2 k x)-16 f(k x)+\left(k-k^{3}\right) f(4 x)+\left(2 k^{3}-10 k\right) f(2 x)+16 k f(x)}(t) \\
\geq\left(\tau_{M}\right)_{i=1}^{23}\left(\mu_{\varphi(x,(k+1) x)}^{\prime}\left(\frac{t}{64}\right), \mu_{\varphi(x,(k-1) x)}^{\prime}\left(\frac{t}{64}\right), \mu_{\varphi(0, x)}^{\prime}\left(\frac{(k-1) t}{64}\right), \mu_{\varphi(0, k x)}^{\prime}\left(\frac{(k-1) t}{64 k}\right),\right. \\
\mu_{\varphi(2 x, x)}^{\prime}\left(\frac{t}{16}\right), \mu_{\varphi(2 x, k x)}^{\prime}\left(\frac{t}{8}\right), \mu_{\varphi(x,(2 k-1) x)}^{\prime}\left(\frac{t}{28}\right), \\
\mu_{\varphi(x,(2 k+1) x)}^{\prime}\left(\frac{t}{28}\right), \mu_{\varphi(3 x, x)}^{\prime}\left(\frac{t}{28}\right), \mu_{\varphi(x, x)}^{\prime}\left(\frac{t}{28}\right), \mu_{\varphi(0,(k+1) x)}^{\prime}\left(\frac{(k-1) t}{28}\right), \\
\mu_{\varphi(0,(k-1) x)}^{\prime}\left(\frac{(k-1) t}{28}\right), \mu_{\varphi(0,2 k x)}^{\prime}\left(\frac{(k-1) t}{28 k}\right), \mu_{\varphi(x,(2 k+1) x)}^{\prime}\left(\frac{t}{192 k}\right), \\
\mu_{\varphi(x,(2 k-1) x)}^{\prime}\left(\frac{t}{192 k}\right), \mu_{\varphi(x, 3 k x)}^{\prime}\left(\frac{t}{192}\right), \mu_{\varphi(0,(3 k-1) x)}^{\prime}\left(\frac{(k-1) t}{192 k}\right), \\
\mu_{\varphi(2 x, 2 x)}^{\prime}\left(\frac{t}{48 k^{2}}\right), \mu_{\varphi(x, k x)}^{\prime}\left(\frac{t}{48}\right), \mu_{\varphi(0,(k+1) x)}^{\prime}\left(\frac{(k-1) t}{48 k}\right) \\
\left.\mu_{\varphi(0,2(k-1) x)}^{\prime}\left(\frac{(k-1) t}{48 k^{2}}\right), \mu_{\varphi(0,2 k x)}^{\prime}\left(\frac{t}{48(k+1)}\right), \mu_{\varphi(2 x, 2 k x)}^{\prime}\left(\frac{t}{8}\right)\right)
\end{gathered}
$$

for all $x \in X$ and $t>0$. Therefore by (3.34) and (3.38), we get

$$
\mu_{f(4 x)-10 f(2 x)+16 f(x)}\left(\frac{t}{k^{3}-k}\right) \geq \psi_{x}(t)
$$


for all $x \in X$ and $t>0$. By Corollary 3.2, there exists a unique mapping $A: X \rightarrow Y$ such that $A(2 x)=2 A(x)$ and $\mu_{f(2 x)-8 f(x)-A(x)}(t) \geq \psi_{x}\left((2-\alpha)\left(k^{3}-k\right) t / 2\right)$ for all $x \in X$ and $t>0$.

It remains to show that $A$ is an additive map. Replacing $x, y$ by $2^{n} x, 2^{n} y$ in (3.12) we get

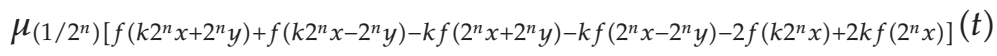

$$
\begin{aligned}
& \geq \mu_{\varphi(x, y)}^{\prime}\left(\frac{2^{n} t}{a^{n}}\right)
\end{aligned}
$$

for all $x, y \in X$ and $t>0$. Hence

$$
\begin{gathered}
\mu_{f(k x+y)+f(k x-y)-k f(x+y)-k f(x-y)-2 f(k x)+2 k f(x)}(t) \\
\geq\left(\tau_{M}\right)_{i=1}^{8}\left(\mu_{A(k x+y)-\left(g\left(2^{n}(k x+y)\right)\right) / 2^{n}}\left(\frac{t}{8}\right), \mu_{A(k x-y)-\left(g\left(2^{n}(k x-y)\right)\right) / 2^{n}}\left(\frac{t}{8}\right),\right. \\
\mu_{A(x+y)-\left(g\left(2^{n}(x+y)\right)\right) / 2^{n}}\left(\frac{t}{8 k}\right), \mu_{A(x-y)-\left(g\left(2^{n}(x-y)\right)\right) / 2^{n}}\left(\frac{t}{8 k}\right), \\
\mu_{A(k x)-\left(g\left(2^{n} k x\right)\right) / 2^{n}}\left(\frac{t}{16}\right), \mu_{A(x)-\left(g\left(2^{n} x\right)\right) / 2^{n}}\left(\frac{t}{16 k}\right), \\
\left.\mu_{\varphi(x, y)}^{\prime}\left(\frac{2^{n} t}{8 \alpha^{n+1}}\right), \mu_{\varphi(x, y)}^{\prime}\left(\frac{2^{n} t}{64 \alpha^{n}}\right)\right)
\end{gathered}
$$

for all $x, y \in X$ and $t>0$. Taking the limit as $n \rightarrow \infty$ in (3.41), we conclude that $A$ fulfills (1.2), and so by [16, Lemma 3.1], we see that the mapping $x \rightarrow A(2 x)-8 A(x)$ is additive, which implies that the mapping $A$ is additive. This completes the proof.

Similar to Theorem 3.3, one can prove the following result.

Theorem 3.4. Let $X$ be a linear space, $\left(Z, \mu^{\prime}, \tau_{M}\right)$ be an $R N$-space, $\left(Y, \mu, \tau_{M}\right)$ be a complete $R N$ space, and $f: X \rightarrow Y$ be a mapping with $f(0)=0$ for which there is $\varphi: X \times X \rightarrow Z$ such that

$$
\mu_{f(k x+y)+f(k x-y)-k f(x+y)-k f(x-y)-2 f(k x)+2 k f(x)}(t) \geq \mu_{\varphi(x, y)}^{\prime}(t)
$$

for all $x, y \in X$ and $t>0$. If for some $0<\alpha<8, \mu_{\varphi(2 x, 2 y)}^{\prime}(t) \geq \mu_{\alpha \varphi(x, y)}^{\prime}(t)$ for all $x, y \in X$ and $t>0$, then there exists a unique cubic mapping $C: X \rightarrow Y$ such that

$$
\mu_{f(2 x)-2 f(x)-C(x)}(t) \geq \psi_{x}\left(\frac{(8-\alpha)\left(k^{3}-k\right) t}{2}\right)
$$

for all $x \in X$ and $t>0$, where $\psi_{x}(t)$ is defined as in Theorem 3.3.

Remark 3.5. We can also prove Theorems 3.3 and 3.4 for $\alpha>2$ and $\alpha>8$, respectively. 
Theorem 3.6. Let $X$ be a linear space, $\left(Z, \mu^{\prime}, \tau_{M}\right)$ be an $R N$-space, $\left(Y, \mu, \tau_{M}\right)$ be a complete $R N$ space, and $f: X \rightarrow Y$ be a mapping with $f(0)=0$ for which there is $\varphi: X \times X \rightarrow Z$ such that

$$
\mu_{f(k x+y)+f(k x-y)-k f(x+y)-k f(x-y)-2 f(k x)+2 k f(x)}(t) \geq \mu_{\varphi(x, y)}^{\prime}(t)
$$

for all $x, y \in X$ and $t>0$. If for some $0<\alpha<2$,

$$
\mu_{\varphi(2 x, 2 y)}^{\prime}(t) \geq \mu_{\alpha \varphi(x, y)}^{\prime}(t)
$$

for all $x, y \in X$ and $t>0$, then there exist a unique additive mapping $A: X \rightarrow Y$ and a unique cubic mapping $C: X \rightarrow Y$ such that

$$
\mu_{f(x)-A(x)-C(x)}(t) \geq \psi_{x}\left(\frac{3(2-\alpha)\left(k^{3}-k\right) t}{2}\right)
$$

for all $x \in X$ and $t>0$, where $\psi_{x}(t)$ is defined as in Theorem 3.3 .

Proof. By Theorems 3.3 and 3.4, there exist an additive mapping $A_{1}: X \rightarrow Y$ and a cubic mapping $C_{1}: X \rightarrow Y$ such that

$$
\begin{aligned}
& \mu_{f(2 x)-8 f(x)-A_{1}(x)}(t) \geq \psi_{x}\left(\frac{(2-\alpha)\left(k^{3}-k\right) t}{2}\right), \\
& \mu_{f(2 x)-2 f(x)-C_{1}(x)}(t) \geq \psi_{x}\left(\frac{(8-\alpha)\left(k^{3}-k\right) t}{2}\right)
\end{aligned}
$$

for all $x \in X$ and $t>0$. Therefore from (3.47) and (3.48), we get

$$
\mu_{f(x)+\left(\frac{1}{6}\right) A_{1}(x)-\left(\frac{1}{6}\right) C_{1}(x)}(t) \geq \psi_{x}\left(\frac{3(2-\alpha)\left(k^{3}-k\right) t}{2}\right)
$$

for all $x \in X$ and $t>0$. Letting $A(x)=-(1 / 6) A_{1}(x)$ and $C(x)=(1 / 6) C_{1}(x)$ for all $x \in X$, it follows from (3.49) that

$$
\mu_{f(x)-A(x)-C(x)}(t) \geq \psi_{x}\left(\frac{3(2-\alpha)\left(k^{3}-k\right) t}{2}\right)
$$


for all $x \in X$ and $t>0$. To prove the uniqueness of $A$ and $C$, let $A^{\prime}, C^{\prime}: X \rightarrow Y$ be another additive and cubic mapping satisfying (3.46). Set $\tilde{A}=A-A^{\prime}$ and $\tilde{C}=C-C^{\prime}$. So

$$
\begin{aligned}
\mu_{\tilde{A}(x)+\tilde{C}(x)}(t) & \geq \tau_{M}\left(\mu_{A(x)+C(x)-f(x)}\left(\frac{t}{2}\right), \mu_{f(x)-A^{\prime}(x)-C^{\prime}(x)}\left(\frac{t}{2}\right)\right) \\
& \geq \psi_{x}\left(\frac{3(2-\alpha)\left(k^{3}-k\right) t}{4}\right)
\end{aligned}
$$

for all $x \in X$ and $t>0$. By $\widetilde{A}(2 x)=2 \widetilde{A}(x), \widetilde{C}(2 x)=8 \tilde{C}(x)$, and (3.51), we get

$$
\begin{aligned}
\mu_{\tilde{C}(x)}(t) & \geq \tau_{M}\left(\mu_{\tilde{A}\left(2^{n} x\right)+\tilde{C}\left(2^{n} x\right)}\left(\frac{8^{n} t}{2}\right), \mu_{\tilde{A}\left(2^{n} x\right)}\left(\frac{8^{n} t}{2}\right)\right) \\
& \geq \tau_{M}\left(\psi_{x}\left(\frac{3(2-\alpha)\left(k^{3}-k\right) 8^{n} t}{4 \alpha^{n}}\right), \mu_{\tilde{A}(x)}\left(\frac{4^{n} t}{2}\right)\right)
\end{aligned}
$$

for all $x \in X$ and $t>0$. Since the right hand side of the inequality tends to 1 as $n$ tend to infinity, we find that $\tilde{C}(x)=0$. Therefore $\widetilde{C}=0$, and then $\widetilde{A}=0$. This completes the proof.

Remark 3.7. We can formulate similar statements to Theorem 3.6 for $\alpha>8$.

Corollary 3.8. Let $(X,\|\cdot\|)$ be a normed space, $\left(Z, \mu^{\prime}, \tau_{M}\right)$ be an $R N$-space, and $\left(Y, \mu, \tau_{M}\right)$ be a complete RN-space. Let $p$ be a non-negative real number such that $p \in(0,1) \cup(1,3) \cup(3, \infty)$, and let $z_{0} \in Z$. If $f: X \rightarrow Y$ is a mapping with $f(0)=0$ such that

$$
\mu_{f(k x+y)+f(k x-y)-k f(x+y)-k f(x-y)-2 f(k x)+2 k f(x)}(t) \geq \mu_{\left(\|x\|^{p}+\|y\|^{p}\right) z_{0}}^{\prime}(t)
$$

for all $x, y \in X$ and $t>0$, then there exist a unique additive mapping $A: X \rightarrow Y$ and a unique cubic mapping $C: X \rightarrow Y$ such that

$$
\mu_{f(x)-A(x)-C(x)}(t) \geq \begin{cases}\mu_{\|x\|^{p} z_{0}}^{\prime}\left(\frac{\left(2-2^{p}\right)\left(k^{2}-1\right) t}{256[1+3 k]}\right), & p \in(0,1), \\ \mu_{\|x\|^{p} z_{0}}^{\prime}\left(\frac{\left(2^{p}-2\right)\left(k^{2}-1\right) t}{256\left[1+(3 k)^{3}\right]}\right), & p \in\left(1, \log _{2} 5\right), \\ \mu_{\|x\|^{p} z_{0}}^{\prime}\left(\frac{\left(8-2^{p}\right)\left(k^{2}-1\right) t}{256\left[1+(3 k)^{3}\right]}\right), & p \in\left(\log _{2} 5,3\right), \\ \mu_{\|x\|^{p} z_{0}}^{\prime}\left(\frac{\left(2^{p}-8\right)\left(k^{2}-1\right) t}{256\left[1+(3 k)^{p}\right]}\right), & p \in(3, \infty),\end{cases}
$$

for all $x \in X$ and $t>0$. 
Corollary 3.9. Let $(X,\|\cdot\|)$ be a normed space, $\left(Z, \mu^{\prime}, \tau_{M}\right)$ be an $R N$-space, and $\left(Y, \mu, \tau_{M}\right)$ be a complete RN-space. Let $r$, s be non-negative real numbers such that $\lambda:=r+s \in(0,1) \cup(1,3) \cup(3, \infty)$, and let $z_{0} \in Z$. If $f: X \rightarrow Y$ be a mapping with $f(0)=0$ such that

$$
\mu_{f(k x+y)+f(k x-y)-k f(x+y)-k f(x-y)-2 f(k x)+2 k f(x)}(t) \geq \mu_{\left[\|x\|^{r}\|y\|^{s}+\left(\|x\|^{r+s}+\|y\|^{r+s}\right)\right] z_{0}}^{\prime}(t)
$$

for all $x, y \in X$ and $t>0$, then there exist a unique additive mapping $A: X \rightarrow Y$ and a unique cubic mapping $C: X \rightarrow Y$ such that

$$
\mu_{f(x)-A(x)-C(x)}(t) \geq \begin{cases}\mu_{\|x\|^{\lambda} z_{0}}^{\prime}\left(\frac{\left(2-2^{\lambda}\right)\left(k^{2}-1\right) t}{256[1+6 k]}\right), & \lambda \in(0,1), \\ \mu_{\|x\|^{\prime} z_{0}}^{\prime}\left(\frac{\left(2^{\lambda}-2\right)\left(k^{2}-1\right) t}{256\left[1+2(3 k)^{3}\right]}\right), & \lambda \in\left(1, \log _{2} 5\right), \\ \mu_{\|x\|^{\prime} z_{0}}^{\prime}\left(\frac{\left(8-2^{\lambda}\right)\left(k^{2}-1\right) t}{256\left[1+2(3 k)^{3}\right]}\right), & \lambda \in\left(\log _{2} 5,3\right), \\ \mu_{\|x\|^{\prime} z_{0}}^{\prime}\left(\frac{\left(2^{\lambda}-8\right)\left(k^{2}-1\right) t}{256\left[1+2(3 k)^{\lambda}\right]}\right), & \lambda \in(3, \infty),\end{cases}
$$

for all $x \in X$ and $t>0$.

Now, we give one example to illustrate the main results of Theorem 3.6. This example is a modification of the example of Zhang et al. [34].

Example 3.10. Let $(X,\|\cdot\|)$ be a Banach algebra, $x_{0}$ be a unit vector in $X$, and $\mu_{x}(t)$ is defined as in Example 2.3. It is easy to see that $\left(X, \mu, \tau_{M}\right)$ is a complete $R N$-space.

Define $f: X \rightarrow X$ by $f(x)=x^{3}+\|x\|^{p} x_{0}$ for $x \in X$. For $0<p<1$, define

$$
\varphi(x, y)=8 k\left(\|x\|^{p}+\|y\|^{p}\right) x_{0}, \quad x, y \in X .
$$

Since $0<p<1$, the inequality $(a+b)^{p} \leq a^{p}+b^{p}$ holds when $a \geq 0$ and $b \geq 0$. A straightforward computation shows that

$$
\|f(k x+y)+f(k x-y)-k f(x+y)-k f(x-y)-2 f(k x)+2 k f(x)\| \leq 8 k\left(\|x\|^{p}+\|y\|^{p}\right)
$$


for all $x, y \in X$. Therefore, all the conditions of Theorem 3.6 hold, and there exist a unique additive mapping $A: X \rightarrow X$ and a unique cubic mapping $C: X \rightarrow X$ such that

$$
\mu_{f(x)-A(x)-C(x)}(t) \geq \psi_{x}\left(\frac{3(2-\alpha)\left(k^{3}-k\right) t}{2}\right)
$$

for all $x \in X$ and $t>0$, where $\psi_{x}(t)$ is defined as in Theorem 3.3.

\section{Acknowledgments}

The authors would like to thank the area editor professor Radu Precup and two anonymous referees for their valuable comments and suggestions. T. Z. Xu was supported by the National Natural Science Foundation of China (10671013).

\section{References}

[1] Z. Moszner, "On the stability of functional equations," Aequationes Mathematicae, vol. 77, no. 1-2, pp. 33-88, 2009.

[2] S. M. Ulam, A Collection of Mathematical Problems, Interscience Tracts in Pure and Applied Mathematics, Interscience Publishers, New York, NY, USA, 1960.

[3] D. H. Hyers, "On the stability of the linear functional equation," Proceedings of the National Academy of Sciences of the United States of America, vol. 27, pp. 222-224, 1941.

[4] Th. M. Rassias, "On the stability of the linear mapping in Banach spaces," Proceedings of the American Mathematical Society, vol. 72, no. 2, pp. 297-300, 1978.

[5] P. Găvruta, "A generalization of the Hyers-Ulam-Rassias stability of approximately additive mappings," Journal of Mathematical Analysis and Applications, vol. 184, no. 3, pp. 431-436, 1994.

[6] W. Fechner, "On the Hyers-Ulam stability of functional equations connected with additive and quadratic mappings," Journal of Mathematical Analysis and Applications, vol. 322, no. 2, pp. 774-786, 2006.

[7] S. M. Jung, "On the Hyers-Ulam stability of the functional equations that have the quadratic property," Journal of Mathematical Analysis and Applications, vol. 222, no. 1, pp. 126-137, 1998.

[8] J. Sikorska, "On a direct method for proving the Hyers-Ulam stability of functional equations," Journal of Mathematical Analysis and Applications, vol. 372, no. 1, pp. 99-109.

[9] J. Brzdȩk, "On a method of proving the Hyers-Ulam stability of functional equations on restricted domains," The Australian Journal of Mathematical Analysis and Applications, vol. 6, no. 1, article no. 4, p. 10, 2009.

[10] J. Brzdęk and A. Pietrzyk, "A note on stability of the general linear equation," Aequationes Mathematicae, vol. 75, no. 3, pp. 267-270, 2008.

[11] G.-L. Forti, "Comments on the core of the direct method for proving Hyers-Ulam stability of functional equations," Journal of Mathematical Analysis and Applications, vol. 295, no. 1, pp. 127-133, 2004.

[12] G.-L. Forti, "Elementary remarks on Ulam-Hyers stability of linear functional equations," Journal of Mathematical Analysis and Applications, vol. 328, no. 1, pp. 109-118, 2007.

[13] K. Ravi, M. Arunkumar, and J. M. Rassias, "Ulam stability for the orthogonally general EulerLagrange type functional equation," International Journal of Mathematics and Statistics, vol. 3, no. A06, pp. 36-46, 2008.

[14] T. Z. Xu, J. M. Rassias, and W. X. Xu, "Stability of a general mixed additive-cubic functional equation in non-Archimedean fuzzy normed spaces," Journal of Mathematical Physics, vol. 51, 19 pages, 2010.

[15] T. Z. Xu, J. M. Rassias, and W. X. Xu, "A fixed point approach to the stability of a general mixed additive-cubic functional equation in quasi fuzzy normed spaces," to appear in International Journal of Physical Sciences.

[16] T. Z. Xu, J. M. Rassias, and W. X. Xu, "Intuitionistic fuzzy stability of a general mixed additive-cubic equation," Journal of Mathematical Physics, vol. 51, no. 6, 21 pages, 2010. 
[17] M. Mohamadi, Y. J. Cho, C. Park, P. Vetro, and R. Saadati, "Random stability of an additive-quadraticquartic functional equation," Journal of Inequalities and Applications, vol. 2010, Article ID 754210, 18 pages, 2010.

[18] E. Baktash, Y. J. Cho, M. Jalili, R. Saadati, and S. M. Vaezpour, "On the stability of cubic mappings and quadratic mappings in random normed spaces," Journal of Inequalities and Applications, vol. 2008, Article ID 902187, 11 pages, 2008.

[19] L. Cădariu and V. Radu, "Fixed points and stability for functional equations in probabilistic metric and random normed spaces," Fixed Point Theory and Applications, vol. 2009, Article ID 589143, 18 pages, 2009.

[20] M. Eshaghi Gordji and M. B. Savadkouhi, "Stability of mixed type cubic and quartic functional equations in random normed spaces," Journal of Inequalities and Applications, vol. 2009, Article ID 527462, 9 pages, 2009.

[21] M. Gordji Eshaghi, J. M. Rassias, and M. B. Savadkouhi, "Approximation of the quadratic and cubic functional equations in RN-spaces," European Journal of Pure and Applied Mathematics, vol. 2, no. 4, pp. 494-507, 2009.

[22] D. Miheț and V. Radu, “On the stability of the additive Cauchy functional equation in random normed spaces," Journal of Mathematical Analysis and Applications, vol. 343, no. 1, pp. 567-572, 2008.

[23] D. Miheţ, "The stability of the additive Cauchy functional equation in non-Archimedean fuzzy normed spaces," Fuzzy Sets and Systems, vol. 161, pp. 2206-2212, 2010.

[24] A. K. Mirmostafaee and M. S. Moslehian, "Stability of additive mappings in non-Archimedean fuzzy normed spaces," Fuzzy Sets and Systems, vol. 160, no. 11, pp. 1643-1652, 2009.

[25] M. S. Moslehian and Th. M. Rassias, "Stability of functional equations in non-Archimedean spaces," Applicable Analysis and Discrete Mathematics, vol. 1, no. 2, pp. 325-334, 2007.

[26] M. S. Moslehian, K. Nikodem, and D. Popa, "Asymptotic aspect of the quadratic functional equation in multi-normed spaces," Journal of Mathematical Analysis and Applications, vol. 355, no. 2, pp. 717-724, 2009.

[27] C. Park, "Fixed points and the stability of an AQCQ-functional equation in non-Archimedean normed spaces," Abstract and Applied Analysis, vol. 2010, Article ID 849543, 15 pages, 2010.

[28] R. Saadati, S. M. Vaezpour, and Y. J. Cho, "A note to paper "On the stability of cubic mappings and quartic mappings in random normed spaces"," Journal of Inequalities and Applications, vol. 2009, Article ID 214530, 6 pages, 2009.

[29] A. Najati and G. Z. Eskandani, "Stability of a mixed additive and cubic functional equation in quasiBanach spaces," Journal of Mathematical Analysis and Applications, vol. 342, no. 2, pp. 1318-1331, 2008.

[30] J. Brzdȩk and S.-M. Jung, "A note on stability of a linear functional equation of second order connected with the Fibonacci numbers and Lucas sequences," Journal of Inequalities and Applications.

[31] J. Brzdęk, D. Popa, and B. Xu, "Hyers-Ulam stability for linear equations of higher orders," Acta Mathematica Hungarica, vol. 120, no. 1-2, pp. 1-8, 2008.

[32] S. M. Jung, "Functional equation $f(x)=p f(x-1)-q f(x-2)$ and its Hyers-Ulam stability," Journal of Inequalities and Applications, vol. 2009, Article ID 181678, 10 pages, 2009.

[33] S.-M. Jung, "Hyers-Ulam stability of Fibonacci functional equation," Bulletin of the Iranian Mathematical Society, vol. 35, no. 2, pp. 217-227, 2009.

[34] S.-S. Zhang, J. M. Rassias, and R. Saadati, "Stability of a cubic functional equation in intuitionistic random normed spaces," Journal of Applied Mathematics and Mechanics, vol. 31, no. 1, pp. 21-26, 2010. 